\title{
Neutron production by fast protons from ultraintense laser-plasma interactions
}

\author{
J. M. Yang ${ }^{\text {a) }}$ and P. McKenna ${ }^{\text {b) }}$ \\ Department of Physics, University of Strathclyde, Glasgow, G4 ONG, United Kingdom
}

K. W. D. Ledingham ${ }^{\text {c) }}$

Department of Physics, University of Strathclyde, Glasgow, G4 ONG, United Kingdom and Atomic Weapons Establishment plc Aldermaston, Reading RG7 4PR, United Kingdom

T. McCanny

Department of Physics, University of Strathclyde, Glasgow, G4 ONG, United Kingdom

L. Robson

Department of Physics, University of Strathclyde, Glasgow, G4 ONG, United Kingdom and Atomic Weapons Establishment plc Aldermaston, Reading RG7 4PR, United Kingdom

S. Shimizu ${ }^{\text {d) }}$

Department of Physics, University of Strathclyde, Glasgow, G4 ONG, United Kingdom

R. P. Singhal

Department of Physics and Astronomy, University of Glasgow, Glasgow, G12 8QQ, United Kingdom

M. S. Wei and K. Krushelnick

Blackett Laboratory, Imperial College, London SW7 2BZ, United Kingdom

\author{
R. J. Clarke, D. Neely, and P. A. Norreys \\ Central Laser Facility, Rutherford Appleton Laboratory, Chilton, Didcot, Oxon OX11 OQX, United Kingdom
}

(Received 1 June 2004; accepted 21 September 2004)

Tens of $\mathrm{MeV}$ proton beams have been generated by interactions of the VULCAN petawatt laser with foil targets and used to induce nuclear reactions in zinc and boron samples. The numbers of ${ }^{11} \mathrm{C}$, ${ }^{66} \mathrm{Ga},{ }^{67} \mathrm{Ga},{ }^{68} \mathrm{Ga},{ }^{61} \mathrm{Cu},{ }^{62} \mathrm{Zn},{ }^{63} \mathrm{Zn}$, and ${ }^{69 m} \mathrm{Zn}$ nuclei have been measured and used to determine the proton energy spectrum. It is known that $(p, n)$ reactions provide an important method for producing neutron sources and in the present experiment up to $\sim 10^{9}$ neutrons $\mathrm{sr}^{-1}$ have been generated via ${ }^{11} \mathrm{~B}(p, n){ }^{11} \mathrm{C}$ reactions. Using experimentally determined proton energy spectra, the production of neutrons via $(p, n)$ reactions in various targets has been simulated, to quantify neutron pulse intensities and energy spectra. It has been shown that as high as $4 \times 10^{9}$ neutrons sr ${ }^{-1}$ per laser pulse can be generated via ${ }^{7} \operatorname{Li}(p, n)^{7} \mathrm{~B}$ reactions using the present VULCAN petawatt laser-pulse conditions. (C) 2004 American Institute of Physics. [DOI: 10.1063/1.1814421]

\section{INTRODUCTION}

Laser-solid interactions at intensities greater than $10^{19} \mathrm{~W} / \mathrm{cm}^{2}$ lead to the production of fast electrons with maximum energies greater than $300 \mathrm{MeV},{ }^{1}$ multi-MeV $\gamma$ rays, tens of $\mathrm{MeV}$ protons, ${ }^{2-5}$ and several hundreds of $\mathrm{MeV}$ heavy ions. ${ }^{6-9}$ These energetic particles and high-energy $\gamma$ rays have a number of potential applications, e.g., in the past few years multi-MeV proton beams, generated from intense laser-plasma interactions, have been used to induce nuclear reactions in low- $\mathrm{Z}$ materials such as ${ }^{11} \mathrm{~B}$ and $\mathrm{H}_{2}{ }^{18} \mathrm{O}$, in order to produce short-lived positron emitting isotopes of medical interest. $^{10-12}$ Multi-MeV proton beams have also been used as a particle probe to measure electric charging of microscopic targets irradiated by laser pulses of intensity

\footnotetext{
a)Present address: Research Center of Laser Fusion, P.O. Box 919-986, Mianyang, 621900, P. R. China.

b) Electronic mail: p.mckenna@phys.strath.ac.uk

${ }^{c)}$ Electronic mail: k.ledingham@phys.strath.ac.uk

${ }^{d)}$ Present address: Institute for Chemical Research, Kyoto University, Gokasho, Uji, Kyoto, 611-0011, Japan.
}

$\sim 10^{19} \mathrm{~W} / \mathrm{cm}^{2} .^{13}$ Laser-driven $(\gamma, n)$ and $(\gamma, f)$ reactions have been demonstrated experimentally and used to diagnose plasma parameters $^{14-16}$ and to demonstrate transmutation of long half-life nuclear waste nuclei, such as ${ }^{129} \mathrm{I}^{17,18}$ Heavy ion-induced reactions have also been realized experimentally and the quantities of observed reactions have been increased remarkably by heating the target. ${ }^{8,9}$

The energy spectra of laser-plasma accelerated protons are measured to study proton generation and acceleration mechanisms (Refs. 5 and 19 ). ${ }^{63} \mathrm{Cu}(p, n){ }^{63} \mathrm{Zn}$ reactions in copper stacks are used to quantify the proton numbers through measurement of $\beta^{+}$decay of ${ }^{63} \mathrm{Zn}$ nuclei, using a $\mathrm{NaI}$ detector-based coincidence counting system. As the laser intensity increases, protons are produced with much higher energies ${ }^{5}$ and in addition proton-induced reactions with higher $Q$ values, such as $(p, 2 n),(p, 3 n)$, and $(p, p+n)$ reactions can be produced. Measurement of these proton-induced reactions in a single layer of copper foil has been identified as an alternative method to the use of copper stacks in diagnosing accelerated proton energy spectra from laser-plasma interactions. $^{20}$ 
It is well known that neutrons have a variety of existing and potential applications, such as boron neutron capture therapy (BNCT), neutron radiography, and transmutation of nuclear waste. With respect to BNCT, the two fragments emitted by the boron neutron capture reaction ${ }^{10} \mathrm{~B}(n, \alpha){ }^{7} \mathrm{Li}$ have a range in human tissue that is of the same order of the cell dimensions. Thus they will deliver their energy only to the tumor cells and kill them when a sufficiently large quantity of boron is selectively delivered to the tumor cells to capture the incident neutrons. ${ }^{21}$ Neutron radiography is a very efficient tool for nondestructive testing of materials. ${ }^{22}$ Contrary to $\mathrm{x}$ rays which penetrate light materials and are absorbed by heavy ones, neutrons can easily pass through thick heavy materials but be strongly attenuated by light materials. Thus neutron radiography is similar in application to $\mathrm{x}$-ray radiography and is complementary to the nature of information supplied. Since neutrons are able to distinguish between different isotopes, neutron radiography is an important tool for studies of radioactive materials as well. Fast neutrons can also induce nuclear reactions and have potential use in the transmutation of nuclear waste. The long-lived waste component ${ }^{129} \mathrm{I}$ (15.7 million year half-life), for example, can capture one neutron to produce ${ }^{130} \mathrm{I}$, which has a much shorter half life of $12.4 \mathrm{~h}$, and which decays via $\beta^{-}$ emission to produce the stable nucleus ${ }^{130} \mathrm{Xe}$.

At present there are two types of neutron sources, ${ }^{23}$ accelerator-based and reactor-based sources. The reactorbased neutron sources can provide steady high-flux neutron beams in the range of $10^{7}-10^{15} \mathrm{ncm}^{-2} \mathrm{~s}^{-1}$, but they require very strict radiation shielding, generate nuclear waste, and cannot be sited in some areas due to safety reasons, for example, in hospitals. Most of the high-flux reactors are now operating at around $10^{15} \mathrm{ncm}^{-2} \mathrm{~s}^{-1}$. On the other hand, the accelerator-based neutron sources produce neutron beams with fluxes in the range $10^{6}-10^{13} \mathrm{ncm}^{-2} \mathrm{~s}^{-1}$, although the most recent accelerator-based pulses systems at ISIS (Rutherford Appleton Laboratory) and the SNS (Oak Ridge) will have higher intensities of the order of $10^{16}-10^{17} \mathrm{ncm}^{-2} \mathrm{~s}^{-1}$. Accelerators also provide pulsed neutron beams, for example, the spallation neutron source (SNS) under construction in USA has a specification to generate pulsed neutron beams with pulse widths in the order of microseconds.

However, in the past few years, with the advent of high power lasers, neutrons can also be generated from the laserproduced plasmas. For example, nearly $10^{13}$ DD fusion neutrons were produced in inertial confinement fusion implosion by the Nova laser using a 30-kJ nanosecond laser. ${ }^{24}$ Lancaster et $a l .{ }^{25}$ has studied neutron generation via ${ }^{7} \mathrm{Li}(p, n){ }^{7} \mathrm{Be}$ reactions using the VULCAN $100 \mathrm{TW}$ ps laser facility at intensities up to $2.5 \times 10^{19} \mathrm{~W} \mathrm{~cm}^{-2}$, and obtained up to 3 $\times 10^{8}$ neutrons sr ${ }^{-1}$ per laser shot. Neutron fluxes of $10^{6} \mathrm{sr}^{-1}$ via the $\mathrm{D}(d, n)^{3} \mathrm{He}$ reaction driven by several tens of femtosecond laser pulses has also been reported. ${ }^{26,27}$

In this paper we report on observed proton-induced neutron reactions in zinc and boron using the VULCAN petawatt laser facility. As several proton-induced reactions in zinc have quite different $Q$ values, the proton spectra from both the front and rear of the targets have been determined from the numbers of the observed nuclei, stopping power of

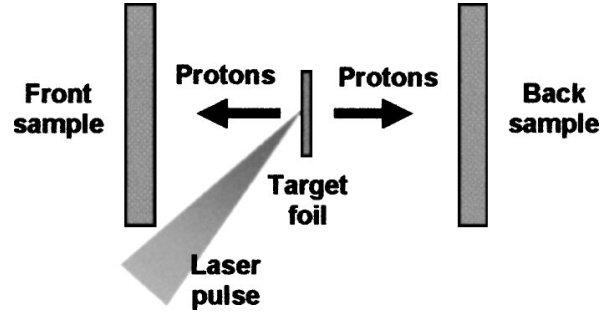

FIG. 1. Schematic experimental arrangement for proton-induced reactions. The ultraintense laser beam was focused on the primary target to generate tens of $\mathrm{MeV}$ protons, which reacted with the samples in front of and behind the target.

the protons in zinc, and reaction cross sections. It was found that as high as $\sim 10^{9}$ neutrons $\mathrm{sr}^{-1}$ have been produced through $(p, n)$ reactions, with a single laser shot. Using the experimentally determined proton spectra, the numbers and energy spectrum of neutrons generated in several different elements have been calculated and discussed.

The potential for laser-driven neutron sources is considerable and offers advantages over accelerator- and reactordriven sources. Firstly, these sources are bright in intensity and since they are produced from very short pulses, timeresolved neutron irradiation experiments become feasible. Although the experiments reported in this paper employ a large petawatt laser, the future in this technology lies in compact high repetition rate, petawatt lasers which are now being constructed in a number of laboratories worldwide. This could potentially permit neutron experiments to be carried out in hospitals and university laboratories with much reduced shielding problems and without access to an accelerator or reactor.

\section{EXPERIMENTAL ARRANGEMENTS}

The experiments were carried out on the petawatt arm of the VULCAN Nd: glass laser at the Rutherford Appleton Laboratory, UK. The $60-\mathrm{cm}$ beam was focused to a 7.0 - $\mu \mathrm{m}$-diam spot using a $1.8-\mathrm{m}$ focal length off-axis parabolic mirror, in a vacuum chamber evacuated to $\sim 10^{-4}$ mbar. The pulse durations were $\sim 750$ fs and the energies on target were 200-300 J for most shots in this experiment. The peak intensities were of the order of 2 to 3 $\times 10^{20} \mathrm{~W} \mathrm{~cm}^{-2}$.

As shown in Fig. 1, primary targets of Mylar, gold, and aluminum foils of various thicknesses were irradiated by the $p$-polarized laser beam at an angle of $45^{\circ}$, to generate multi$\mathrm{MeV}$ proton beams in front of and behind the target foil. Activation samples, such as zinc and boron, were positioned on both sides along the target normal direction. The distance between the target and samples was $38 \mathrm{~mm}$. The samples were exposed to the proton beams from the target and various isotopes were produced via nuclear reactions in the samples. The 100- $\mu$ m-thick zinc samples were analyzed before and after each laser shot using well-shielded germanium detectors. The isotopes and corresponding reactions in the samples were identified based on their emitted $\gamma$-ray energies, intensities, and half-lives. The detection efficiencies of the two germanium detectors were calibrated using known sources of ${ }^{152} \mathrm{Eu},{ }^{57} \mathrm{Co},{ }^{22} \mathrm{Na},{ }^{137} \mathrm{Cs}$, and ${ }^{60} \mathrm{Co}$ to determine 

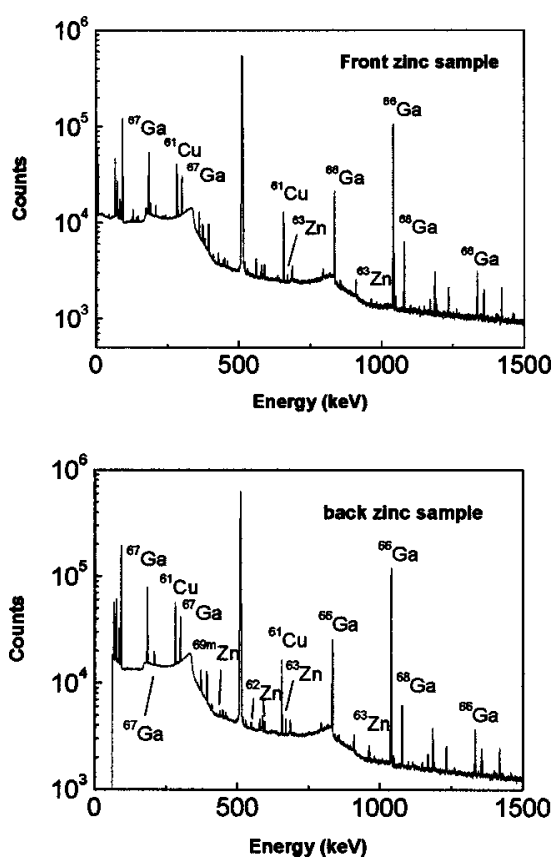

FIG. 2. Regions of characteristic $\gamma$-ray line spectra for the proton-irradiated zinc samples, measured with germanium detectors. Typical lines for the nuclei have been labeled in the spectra.

the absolute numbers of laser-induced reactions. The activity from ${ }^{11} \mathrm{~B}(p, n){ }^{11} \mathrm{C}$ reactions in each boron sample was measured to obtain the number of ${ }^{11} \mathrm{C}$ nuclei and hence the neutron numbers. ${ }^{11} \mathrm{C}$ is a positron emitting source and positrons slow down in solids and annihilate at rest with electrons producing two photons of $511 \mathrm{keV}$ at $180^{\circ}$ to each other. These annihilation photons were detected using two $3 \mathrm{in}$. $\times 3$ in. NaI scintillation detectors setup to detect $511-\mathrm{keV}$ photons and operated in coincidence. ${ }^{5}$ The purity of the source was extremely high and was monitored by measuring the half-life. Any contribution from ${ }^{10} \mathrm{C}$ with a half-life of $20 \mathrm{~s}$ is negligible since the measurements were initiated more than $1 \mathrm{~h}$ after the source was irradiated. The detection efficiency of the NaI coincidence system was also calibrated using the ${ }^{22} \mathrm{Na}$ source.

\section{RESULTS AND DISCUSSION}

The zinc samples positioned in front of and behind the laser-irradiated alumin target were $100-\mu$ m-thick 50 $\times 50 \mathrm{~mm}$ plates. A $20-\mu \mathrm{m}$-thick aluminum primary target was irradiated by a 230-J laser pulse. Regions of the measured $\gamma$-ray spectra from the front and back zinc samples are shown in Fig. 2. Gamma emission from the nuclei ${ }^{66} \mathrm{Ga}$, ${ }^{67} \mathrm{Ga},{ }^{68} \mathrm{Ga},{ }^{61} \mathrm{Cu},{ }^{62} \mathrm{Zn},{ }^{63} \mathrm{Zn}$, and ${ }^{69 \mathrm{~m}} \mathrm{Zn}$ have been identified. The numbers of these nuclei and the corresponding reactions are listed in Table I. As shown in Fig. 2 and Table I, the nuclei ${ }^{66} \mathrm{Ga},{ }^{67} \mathrm{Ga},{ }^{68} \mathrm{Ga},{ }^{61} \mathrm{Cu}$, and ${ }^{63} \mathrm{Zn}$ have been observed in both the front and back zinc samples, but the nuclei ${ }^{62} \mathrm{Zn}$ and ${ }^{69 \mathrm{~m}} \mathrm{Zn}$ have been seen only in the back sample. This is consistent with the following experimentally determined proton spectra, the back proton spectrum has a larger highenergy component than the front one. Since natural zinc has five stable isotopes: ${ }^{64} \mathrm{Zn}(48.6 \%),{ }^{66} \mathrm{Zn}(27.9 \%)$, ${ }^{67} \mathrm{Zn}(4.1 \%),{ }^{68} \mathrm{Zn}(18.8 \%)$, and ${ }^{70} \mathrm{Zn}(0.6 \%)$; after irradiation by the proton beams from the aluminum target, the ${ }^{66} \mathrm{Ga}$ nuclei generated in the samples can result from the three reactions $\quad{ }^{66} \mathrm{Zn}(p, n){ }^{66} \mathrm{Ga}$, $\quad{ }^{67} \mathrm{Zn}(p, 2 n){ }^{66} \mathrm{Ga}$, and ${ }^{68} \mathrm{Zn}(p, 3 n){ }^{66} \mathrm{Ga}$. The ${ }^{67} \mathrm{Ga}$ nuclei could be generated via ${ }^{67} \mathrm{Zn}(p, n){ }^{67} \mathrm{Ga}$ and ${ }^{68} \mathrm{Zn}(p, 2 n){ }^{67} \mathrm{Ga}$ reactions, and the ${ }^{68} \mathrm{Ga}$, ${ }^{61} \mathrm{Cu},{ }^{62} \mathrm{Zn},{ }^{63} \mathrm{Zn}$, and ${ }^{69 m} \mathrm{Zn}$ nuclei were produced via ${ }^{68} \mathrm{Zn}(p, n){ }^{68} \mathrm{Ga}, \quad{ }^{64} \mathrm{Zn}(p, \alpha){ }^{61} \mathrm{Cu}, \quad{ }^{64} \mathrm{Zn}(p, p+2 n)^{62} \mathrm{Zn}$, ${ }^{64} \mathrm{Zn}(p, p+n){ }^{63} \mathrm{Zn}$, and ${ }^{70} \mathrm{Zn}(p, p+n){ }^{69 \mathrm{~m}} \mathrm{Zn}$ reactions, respectively.

As shown in Table I, the proton-induced reactions in zinc have quite different $Q$ values and cross-section peak energies. A code was developed to unfold the proton spectra using the measured numbers of the observed isotopes in zinc. ${ }^{5,19}$ The first step involved the derivation of a response function. The number of nuclei produced by one proton with energy $E_{p}$ was calculated for each reaction using the published proton stopping power and cross-section data. ${ }^{28}$ Then, starting with an arbitrary initial proton spectrum, the number of nuclei for each reaction was calculated by convoluting the spectrum with each response function. The ratio of the measured number of nuclei to calculated value was determined for each nucleus and the proton spectrum was adjusted repeatedly according to the ratio and its corresponding response energy region until the calculated numbers for all observed nuclei were consistent with the measured ones within a preset error. The deduced proton spectra are shown in Fig. 3. Using these spectra, the numbers of the ${ }^{66} \mathrm{Ga}$ nuclei generated from the ${ }^{66} \mathrm{Zn}(p, n){ }^{66} \mathrm{Ga},{ }^{67} \mathrm{Zn}(p, 2 n){ }^{66} \mathrm{Ga}$, and

TABLE I. Residual nuclei observed in the zinc activation target and corresponding proton-induced reactions.

\begin{tabular}{cccccc}
\hline \hline & \multicolumn{2}{c}{ Observed number of nuclei per shot } & & \\
\cline { 2 - 3 } Nuclei & Front & Back & Reactions & $Q$ values $(\mathrm{MeV})$ \\
\hline${ }^{66} \mathrm{Ga}$ & $(2.2 \pm 0.1) \times 10^{8}$ & $(2.2 \pm 0.3) \times 10^{8}$ & ${ }^{66} \mathrm{Zn}(p, n){ }^{66} \mathrm{Ga}$ & -5.957 \\
& & & ${ }^{67} \mathrm{Zn}(p, 2 n){ }^{66} \mathrm{Ga}$ & -13.01 \\
& & & ${ }^{68} \mathrm{Zn}(p, 3 n)^{66} \mathrm{Ga}$ & -23.21 \\
${ }^{67} \mathrm{Ga}$ & $(1.2 \pm 0.1) \times 10^{8}$ & $(1.1 \pm 0.1) \times 10^{8}$ & & ${ }^{67} \mathrm{Zn}(p, n)^{67} \mathrm{Ga}$ & -1.782 \\
& & & ${ }^{68} \mathrm{Zn}(p, 2 n)^{67} \mathrm{Ga}$ & -11.98 \\
${ }^{68} \mathrm{Ga}$ & $(3.0 \pm 0.3) \times 10^{8}$ & $(2.7 \pm 0.3) \times 10^{8}$ & ${ }^{68} \mathrm{Zn}(p, n)^{68} \mathrm{Ga}$ & -3.703 \\
${ }^{61} \mathrm{Cu}$ & $(4.2 \pm 0.1) \times 10^{7}$ & $(4.2 \pm 0.8) \times 10^{7}$ & ${ }^{64} \mathrm{Zn}(p, \alpha){ }^{61} \mathrm{Cu}$ & 0.844 \\
${ }^{62} \mathrm{Zn}$ & $\cdots$ & $(1.2 \pm 0.4) \times 10^{6}$ & ${ }^{64} \mathrm{Zn}(p, p+2 n)^{62} \mathrm{Zn}$ & -20.974 \\
${ }^{63} \mathrm{Zn}$ & $(2.5 \pm 0.8) \times 10^{7}$ & $(5.6 \pm 0.5) \times 10^{7}$ & ${ }^{64} \mathrm{Zn}(p, p+n)^{63} \mathrm{Zn}$ & -11.861 \\
${ }^{69 \mathrm{~m}} \mathrm{Zn}$ & $\cdots$ & $(1.6 \pm 0.3) \times 10^{5}$ & ${ }^{70} \mathrm{Zn}(p, p+n){ }^{69 \mathrm{~m}} \mathrm{Zn}$ & -9.215 \\
\hline \hline
\end{tabular}




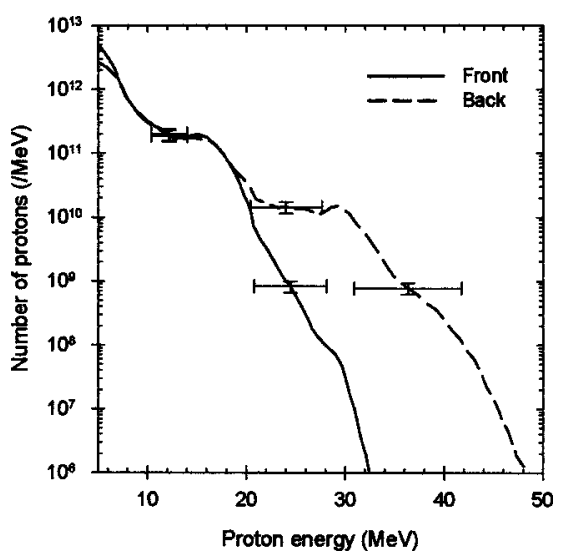

FIG. 3. Proton energy spectra measured at the front and back of a $20-\mu \mathrm{m}$-thick aluminium target foil irradiated with a 230-J laser pulse. The spectra were deduced from observed proton-induced reactions in zinc samples and are in good agreement with proton energy spectra measured with other VULCAN petawatt laser shots and using nuclear activation of $\mathrm{Cu}$ foil stacks (see Refs. 9 and 20). The total conversion efficiency from laserpulse energy to proton acceleration (in both directions) is $\sim 13 \%$. This was calculated by integrating the spectrum in the figure to determine the total energy in the ion beam and dividing this by the energy in the laser shot. The $13 \%$ value is typical for large, high-energy pulse laser (see Ref. 4).

${ }^{68} \mathrm{Zn}(p, 3 n){ }^{66} \mathrm{Ga}$ reactions were calculated. For the front zinc sample, the numbers of ${ }^{66} \mathrm{Ga}$ nuclei from ${ }^{66} \mathrm{Zn}(p, n){ }^{66} \mathrm{Ga}$, ${ }^{67} \mathrm{Zn}(p, 2 n){ }^{66} \mathrm{Ga}$, and ${ }^{68} \mathrm{Zn}(p, 3 n){ }^{66} \mathrm{Ga}$ reactions are $2 \times 10^{8}$, $3 \times 10^{6}$, and $2 \times 10^{3}$, respectively, and for the back sample, these are $2 \times 10^{8}, 5 \times 10^{6}$, and $5 \times 10^{5}$. Therefore, more than $95 \%$ of the ${ }^{66} \mathrm{Ga}$ in the zinc samples came from ${ }^{66} \mathrm{Zn}(p, n){ }^{66} \mathrm{Ga}$ reactions. A similar analysis has been carried out for the generation of the ${ }^{67} \mathrm{Ga}$ nucleus and the results show that, for the front zinc sample, $74 \%$ of ${ }^{67} \mathrm{Ga}$ was produced via ${ }^{67} \mathrm{Zn}(p, n){ }^{67} \mathrm{Ga}$ reactions and $26 \%$ from ${ }^{68} \mathrm{Zn}(p, 2 n){ }^{67} \mathrm{Ga}$, but for the back sample, these figures were $60 \%$ and $40 \%$, respectively, due to the larger fraction of the high-energy proton component in the proton beam at the back of the target.

The measured numbers of ${ }^{11} \mathrm{C}$ nuclei both in the front and back boron samples for different laser-irradiated targets are listed in Table II. The boron samples were 3-mm-thick circular boron disks with a diameter of $50 \mathrm{~mm}$. As shown in Table II, except for the small energy shot, the numbers of the proton-induced ${ }^{11} \mathrm{C}$ nuclei in the front boron samples are almost constant for all shots with this range of target thick-

TABLE II. Observed number of ${ }^{11} \mathrm{C}$ nuclear produced via nuclear reactions induced by proton beams from the front and back of the different laserirradiated targets.

\begin{tabular}{|c|c|c|c|c|}
\hline \multirow[b]{2}{*}{ Target } & \multirow{2}{*}{$\begin{array}{c}\text { Target } \\
\text { thickness } \\
(\mu \mathrm{m})\end{array}$} & \multirow{2}{*}{$\begin{array}{l}\text { Laser } \\
\text { energy } \\
\text { (J) }\end{array}$} & \multicolumn{2}{|c|}{ Observed number of ${ }^{11} \mathrm{C}$ per laser shot } \\
\hline & & & Front sample & Back sample \\
\hline $\mathrm{Al}$ & 1 & 138 & $(3.8 \pm 0.4) \times 10^{9}$ & $(3.7 \pm 0.4) \times 10^{9}$ \\
\hline $\mathrm{Au}$ & 5 & 302 & $(1.3 \pm 0.1) \times 10^{10}$ & $(9.3 \pm 0.9) \times 10^{9}$ \\
\hline $\mathrm{Al}$ & 30 & 214 & $(9.9 \pm 0.9) \times 10^{9}$ & $(4.5 \pm 0.5) \times 10^{9}$ \\
\hline $\mathrm{CH}$ & 75 & 265 & $(7.3 \pm 0.8) \times 10^{9}$ & $(2.8 \pm 0.3) \times 10^{9}$ \\
\hline $\mathrm{Al}$ & 100 & 239 & $(9.4 \pm 0.9) \times 10^{9}$ & $(2.9 \pm 0.3) \times 10^{9}$ \\
\hline $\mathrm{Al}$ & 250 & 243 & $(1.1 \pm 0.1) \times 10^{10}$ & $(3.0 \pm 0.3) \times 10^{9}$ \\
\hline $\mathrm{Al}$ & 500 & 226 & $(9.4 \pm 0.9) \times 10^{9}$ & $(5.6 \pm 0.7) \times 10^{8}$ \\
\hline
\end{tabular}

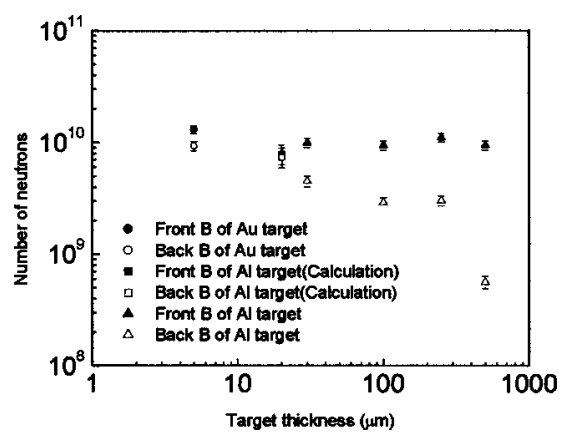

FIG. 4. Numbers of neutrons produced by ${ }^{11} \mathrm{~B}(p, n){ }^{11} \mathrm{C}$ reactions in the front and back boron samples as function of the target thicknesses.

nesses. The small differences in the numbers are reasonable due to the fluctuation of the proton spectrum possibly caused by nonlinear proton generation process ${ }^{5}$ and small changes in the shot-to-shot laser pulse conditions. The number of ${ }^{11} \mathrm{C}$ nuclei in the back boron sample increases with decreasing target thickness for all shots with similar laser energies and tends to be close to the number of ${ }^{11} \mathrm{C}$ in the front sample for the thinnest targets. A description of this data and the analysis is described in detail in Ref. 12.

As each ${ }^{11} \mathrm{~B}(p, n){ }^{11} \mathrm{C}$ reaction generates one neutron and one ${ }^{11} \mathrm{C}$ nucleus, the same number of neutrons are produced as ${ }^{11} \mathrm{C}$ nuclei. Using the proton spectra of Fig. 3, the number of neutrons $N_{n}$, generated in the samples at the front and back of the target can be calculated as follows:

$$
\begin{aligned}
& N_{n}=\int_{E_{\min }}^{E_{\max }} \int_{0}^{x_{0}} n_{i} \sigma\left(E_{p}\right) I_{p}\left(E_{p 0}\right) d E_{p 0} d x, \\
& E_{p}(x)=E_{p 0}-\int_{0}^{x}\left(\frac{d E}{d x}\right) d x_{1},
\end{aligned}
$$

where $x_{0}=\min \left(T_{0}, T_{r}\right)$ is the smaller one of the sample thickness $T_{0}$ and the stopping range $T_{r}$ of the proton of energy $E_{p 0}, n_{i}$ is the number density of the reacting particles in the sample, $I_{p}\left(E_{p 0}\right)$ is the incident proton spectral intensity, $\sigma\left(E_{p}\right)$ is the cross section of the $(p, n)$ reaction, and $d E / d x$ is the stopping power of protons in the sample.

The calculated and observed $\left({ }^{11} \mathrm{C}\right)$ numbers of neutrons for the different targets but similar laser energies are shown in Fig. 4. In the calculations, the ${ }^{11} \mathrm{~B}$ isotopic abundance of $80 \%$ and density of $2.35 \mathrm{~g} \mathrm{~cm}^{-3}$ were adopted. The same sample thicknesses as the experiment were also used. The calculated number of neutrons generated in the front boron sample with the 20- $\mu \mathrm{m}$-thick aluminum target is in good agreement with those observed experimentally (for the range of target thicknesses) and the calculations for the back boron sample also agrees well with the observed reduction of the neutron number with the target thickness, confirming that the proton spectra used in the calculations are realistic. Importantly, up to $10^{9}$ neutrons $\mathrm{sr}^{-1}$ have been produced by a very short pulse $(\sim$ ps $)$ of protons, providing a bright neutron source.

Since $(p, n)$ reactions induced by proton beams from a high intensity laser-plasma interaction is a possible neutron source with many potential applications, it is important and 


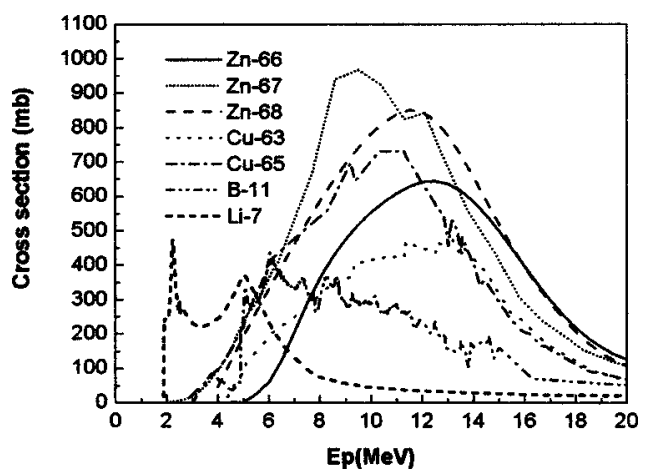

FIG. 5. Cross sections for the $(p, n)$ reactions in ${ }^{7} \mathrm{Li},{ }^{11} \mathrm{~B},{ }^{63} \mathrm{Cu},{ }^{65} \mathrm{Cu},{ }^{66} \mathrm{Zn}$, ${ }^{67} \mathrm{Zn}$, and ${ }^{68} \mathrm{Zn}$ samples (see Ref. 28 ).

necessary to characterize this source, including the neutron intensity, energy spectrum, and the angular distribution of the emitted neutrons. Firstly, to calculate the intensity of neutrons produced via $(p, n)$ reactions, lithium $\left(92.5 \%{ }^{7} \mathrm{Li}\right)$, boron $\left(80 \%{ }^{11} \mathrm{~B}\right), \quad 100 \%{ }^{63} \mathrm{Cu}, \quad 100 \%{ }^{65} \mathrm{Cu}, \quad 100 \%{ }^{66} \mathrm{Zn}$, $100 \%{ }^{67} \mathrm{Zn}$, and $100 \%{ }^{68} \mathrm{Zn}$ have been selected as activation samples. Using $(p, n)$ cross sections ${ }^{28}$ Fig. 5 , and the measured proton energy spectra, the numbers of neutrons as a function of thickness for these samples were calculated using Eqs. (1) and (2) and are presented in Fig. 6. The number of neutrons produced in the sample increases with thickness for a thin sample and tends to become constant as the thickness of the sample increases. It is apparent from Fig. 6 that much thicker low- $Z$ lithium and boron are needed to produce the maximum numbers of neutrons than the medium- $Z$ copper and zinc. This is the result of the much longer proton stopping range in the low- $Z$ samples since the energy loss of a proton in a sample is proportional to the atomic number $Z$ of the sample, in terms of the Bethe formula. Slightly thicker activation samples are required at the back of the target, compared to the front, to maximize neutron production. This is due to the larger numbers of higher-energy protons in the back proton beam (Fig. 3). The calculated maximum numbers of neutrons produced by $(p, n)$ reactions are listed in Table III. For copper, the ${ }^{65} \mathrm{Cu}$ isotope is seen to generate a much larger number of neutrons than ${ }^{63} \mathrm{Cu}$, and for zinc samples, the largest number of neutrons, as high as $\sim 4$ $\times 10^{8} \mathrm{sr}^{-1}$, is produced by reactions on ${ }^{67} \mathrm{Zn}$. This is due to the large cross sections for ${ }^{65} \mathrm{Cu}(p, n){ }^{65} \mathrm{Zn}$ and ${ }^{67} \mathrm{Zn}(p, n){ }^{67} \mathrm{Ga}$ (Fig. 5). Table III also shows that as many as

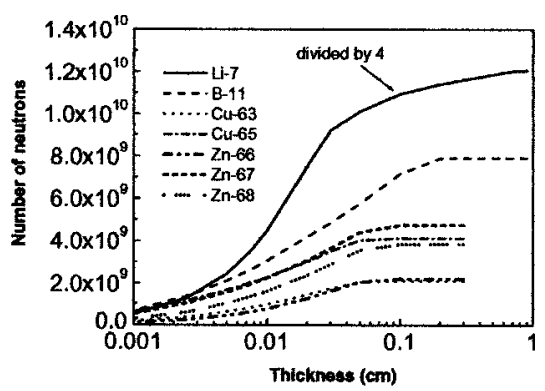

a) Front samples

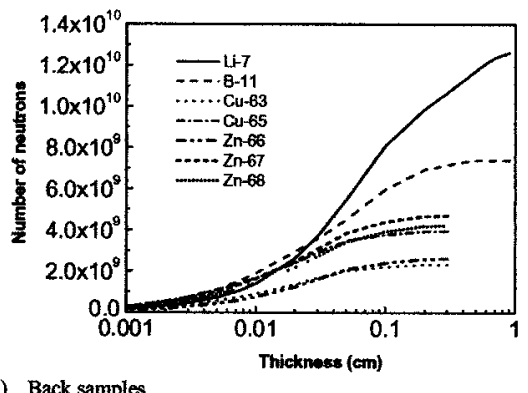

b) Back samples

FIG. 6. Calculated numbers of neutrons generated by $(p, n)$ reactions in the ${ }^{7} \mathrm{Li},{ }^{11} \mathrm{~B},{ }^{63} \mathrm{Cu},{ }^{65} \mathrm{Cu},{ }^{66} \mathrm{Zn},{ }^{67} \mathrm{Zn}$, and ${ }^{68} \mathrm{Zn}$ samples as a function of their thickness for (a) front samples and (b) back samples.

$4 \times 10^{9}$ and $1 \times 10^{9}$ neutrons $\mathrm{sr}^{-1}$ could be produced in front and back lithium samples, respectively. This is larger than is produced in boron samples. ${ }^{11} \mathrm{~B}(p, n){ }^{11} \mathrm{C}$ and ${ }^{7} \mathrm{Li}(p, n){ }^{7} \mathrm{Be}$ reactions have similar peaked reaction cross sections and the cross section for ${ }^{11} \mathrm{~B}(p, n){ }^{11} \mathrm{C}$ has an even broader energy distribution. However, the main part of the ${ }^{7} \operatorname{Li}(p, n)^{7} \mathrm{Be}$ cross section lies in the lower-energy region of the proton spectrum and the number of protons decreases almost exponentially with the increasing proton energy (Fig. 3). The large number of lower-energy protons produce $(p, n)$ reactions in ${ }^{7} \mathrm{Li}$ nuclei and this could account for the much larger number of neutrons in lithium samples, compared to the ${ }^{11} \mathrm{~B}$ sample for which the reaction cross section is peaked at higher energies. In principle, the optimum sample to produce the highest intensity neutrons via $(p, n)$ reactions should have a high reaction cross section (neutron-enriched nucleus) and a long proton stopping range (small atomic number nucleus), the main cross section should also lie in the low-energy part with low reaction threshold.

The energy spectrum is also an important characteristic

TABLE III. Calculated maximum numbers of neutrons per steradian produced by $(p, n)$ reactions in activation samples.

\begin{tabular}{|c|c|c|c|c|c|c|}
\hline \multirow[b]{2}{*}{ Samples } & \multirow{2}{*}{$\begin{array}{c}\text { Densities of } \\
\text { samples } \\
\left(\mathrm{g} / \mathrm{m}^{3}\right)\end{array}$} & \multirow{2}{*}{$\begin{array}{c}\text { Enrichment } \\
\text { of nuclei } \\
(\%)\end{array}$} & \multicolumn{2}{|c|}{$\begin{array}{l}\text { Calculated maximum numbers } \\
\text { of neutrons } \mathrm{sr}^{-1}\end{array}$} & \multirow[b]{2}{*}{ Reactions } & \multirow{2}{*}{$\begin{array}{l}Q \text { values } \\
(\mathrm{MeV})\end{array}$} \\
\hline & & & Front & Back & & \\
\hline${ }^{7} \mathrm{Li}$ & 0.534 & 0.925 & $(3.8 \pm 0.8) \times 10^{9}$ & $(1.0 \pm 0.2) \times 10^{9}$ & ${ }^{7} \mathrm{Li}(p, n){ }^{7} \mathrm{Be}$ & -1.644 \\
\hline${ }^{11} \mathrm{~B}$ & 2.35 & 0.80 & $(6.3 \pm 1.3) \times 10^{8}$ & $(5.9 \pm 1.2) \times 10^{8}$ & ${ }^{11} \mathrm{~B}(p, n){ }^{11} \mathrm{C}$ & -2.765 \\
\hline${ }^{63} \mathrm{Cu}$ & 8.92 & 100 & $(1.7 \pm 0.3) \times 10^{8}$ & $(1.9 \pm 0.4) \times 10^{8}$ & ${ }^{63} \mathrm{Cu}(p, n){ }^{63} \mathrm{Zn}$ & -4.149 \\
\hline${ }^{65} \mathrm{Cu}$ & 8.92 & 100 & $(3.3 \pm 0.7) \times 10^{8}$ & $(3.1 \pm 0.6) \times 10^{8}$ & ${ }^{65} \mathrm{Cu}(p, n){ }^{65} \mathrm{Zn}$ & -2.134 \\
\hline${ }^{66} \mathrm{Zn}$ & 7.14 & 100 & $(1.7 \pm 0.3) \times 10^{8}$ & $(2.1 \pm 0.4) \times 10^{8}$ & ${ }^{66} \mathrm{Zn}(p, n){ }^{66} \mathrm{Ga}$ & -5.957 \\
\hline${ }^{67} \mathrm{Zn}$ & 7.14 & 100 & $(3.8 \pm 0.9) \times 10^{8}$ & $(3.8 \pm 0.8) \times 10^{8}$ & ${ }^{67} \mathrm{Zn}(p, n){ }^{67} \mathrm{Ga}$ & -1.782 \\
\hline${ }^{68} \mathrm{Zn}$ & 7.14 & 100 & $(3.0 \pm 0.6) \times 10^{8}$ & $(3.4 \pm 0.7) \times 10^{8}$ & ${ }^{68} \mathrm{Zn}(p, n){ }^{68} \mathrm{Ga}$ & -3.703 \\
\hline
\end{tabular}




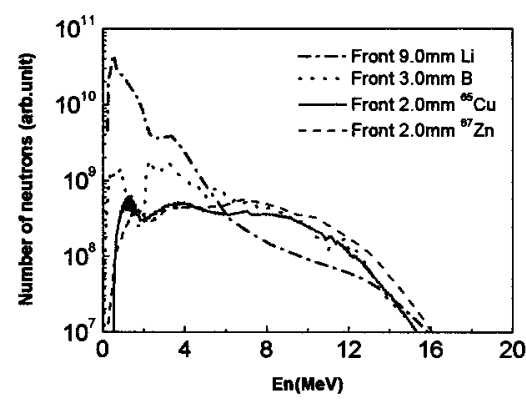

a) Front samples

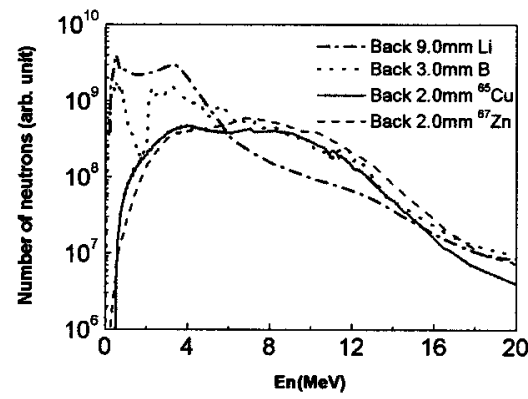

b) Back samples

FIG. 7. Calculated neutron energy spectra generated in the forward direction of the proton beams by $(p, n)$ reactions in ${ }^{7} \mathrm{Li},{ }^{11} \mathrm{~B},{ }^{65} \mathrm{Cu}$, and ${ }^{67} \mathrm{Zn}$ samples.

of this neutron source. The neutron energy spectrum produced by $(p, n)$ reactions can be expressed as

$$
\begin{aligned}
I_{n}\left(E_{n}\right) & =\int_{0}^{T_{0}} n_{i} \sigma\left(E_{p}\right) I_{p}\left(E_{p 0}\right) \frac{\partial E_{p 0}}{\partial E_{n}} d x, \\
E_{n}= & {\left[\frac{m_{1} m_{3}}{\left(m_{3}+m_{4}\right)^{2}}+\frac{m_{2} m_{4}}{\left(m_{1}+m_{2}\right)\left(m_{3}+m_{4}\right)}\right] E_{p} } \\
& +\frac{m_{4}}{\left(m_{3}+m_{4}\right)} Q+2\left[\frac{m_{1} m_{2} m_{3} m_{4}}{\left(m_{1}+m_{2}\right)\left(m_{3}+m_{4}\right)^{3}}\right. \\
& \left.\times\left(E_{p}^{2}+\frac{m_{1}+m_{2}}{m_{2}} Q E_{p}\right)\right]^{1 / 2} \cos \theta,
\end{aligned}
$$

where $E_{n}$ is the neutron energy, $\partial E_{p 0} / \partial E_{n}$ is the derivation of the incident proton energy $E_{p 0}$ over energy $E_{n}$ of the neutron produced at a depth of $x$ in the sample and can be calculated through Eqs. (2) and (4). $m_{1}, m_{2}, m_{3}$, and $m_{4}$ are the masses of the incident proton, reacting target particle, produced neutron, and reaction residual particle, respectively. $Q$ is the $Q$ value of the reaction and $\theta$ is the angle of the neutron in the center-of-mass frame.

The energy spectra of neutrons generated in the lithium, boron, ${ }^{65} \mathrm{Cu}$, and ${ }^{67} \mathrm{Zn}$ samples were calculated and are shown in Fig. 7. Activation samples of thickness 9.0, 3.0, 2.0 , and $2.0 \mathrm{~mm}$ were used in the calculation. As we consider the energy spectra of neutrons emitted only in the forward direction (the direction of the incident proton beam), $\theta$ was set as zero in the calculation [the angular distribution of neutrons emitted from $(p, n)$ reactions can be estimated to be anisotropic from the differential cross section ${ }^{29}$ and mainly in the forward direction in our case]. Other parameters such as $Q$ values, densities, and isotopic enrichments are listed in Table III. The proton spectra and cross-section data used in the calculations are shown in Figs. 2 and 3. As shown in Fig. 7 , the neutrons produced in the ${ }^{65} \mathrm{Cu}$ and ${ }^{67} \mathrm{Zn}$ samples have similar energy distributions. The neutron spectra for both front and back ${ }^{65} \mathrm{Cu}$ and ${ }^{67} \mathrm{Zn}$ samples are broad. The neutron intensities remain almost constant in the energy range of $1-12 \mathrm{MeV}$ and then decrease with increasing neutron energy (above $12.0 \mathrm{MeV}$ ). In contrast, most of the neutrons produced in lithium samples have lower energies $(<4 \mathrm{MeV})$ due to the energy distribution of the reaction cross section (Fig. 5). The neutron spectra for boron is similar to the spectra obtained with ${ }^{65} \mathrm{Cu}$ and ${ }^{67} \mathrm{Zn}$ samples in the higher-energy range of $>7 \mathrm{MeV}$, but much larger at lower energies.

\section{CONCLUSIONS}

In summary, using the VULCAN petawatt laser, tens of $\mathrm{MeV}$ protons have been generated and used to induce nuclear reactions in zinc and boron samples. ${ }^{65} \mathrm{Ga},{ }^{67} \mathrm{Ga}$, ${ }^{68} \mathrm{Ga},{ }^{61} \mathrm{Cu},{ }^{62} \mathrm{Zn},{ }^{63} \mathrm{Zn}$, and ${ }^{69 \mathrm{~m}} \mathrm{Zn}$ nuclei were produced by proton-induced reactions on zinc. These observed nuclei were quantified and used to deduce the incident proton energy spectra. ${ }^{68} \mathrm{Ga},{ }^{61} \mathrm{Cu},{ }^{62} \mathrm{Zn},{ }^{63} \mathrm{Zn}$, and ${ }^{69 \mathrm{~m}} \mathrm{Zn}$ were produced via ${ }^{68} \mathrm{Zn}(p, n){ }^{68} \mathrm{Ga}, \quad{ }^{64} \mathrm{Zn}(p, \alpha){ }^{61} \mathrm{Cu}, \quad{ }^{64} \mathrm{Zn}(p, p$ $+2 n)^{62} \mathrm{Zn},{ }^{64} \mathrm{Zn}(p, p+n)^{63} \mathrm{Zn}$, and ${ }^{70} \mathrm{Zn}(p, p+n){ }^{69 \mathrm{~m}} \mathrm{Zn}$ reactions. More than $95 \%$ of the observed ${ }^{66} \mathrm{Ga}$ was produced via ${ }^{66} \mathrm{Zn}(p, n){ }^{66} \mathrm{Ga}$ reactions. However, analysis on the front zinc sample has shown that $74 \%$ of ${ }^{67} \mathrm{Ga}$ was produced via ${ }^{67} \mathrm{Zn}(p, n){ }^{67} \mathrm{Ga}$ reactions and $26 \%$ from ${ }^{68} \mathrm{Zn}(p, 2 n){ }^{67} \mathrm{Ga}$ and for the back sample these figures were $60 \%$ and $40 \%$, respectively. For boron samples, the numbers of the $(p, n)$ reactiongenerated ${ }^{11} \mathrm{C}$ nuclei have been measured for a series of targets. It was shown for the B sample that the number of ${ }^{11} \mathrm{C}$ nuclei produced via $(p, n)$ reactions in the back boron sample decreases uniformly with the increasing target thickness with similar laser energies. For the thinnest targets, the same numbers of ${ }^{11} \mathrm{C}$ nuclei were generated both in the front and back boron samples. If we consider only the front samples, the numbers of the proton-induced reactions are almost the same for all thicknesses of targets for shots with similar laser parameters. The ${ }^{11} \mathrm{~B}(p, n){ }^{11} \mathrm{C}$ reaction is an important reaction for production of the medical isotope ${ }^{11} \mathrm{C}$. As $(p, n)$ reactions also generate neutrons, it could also be used as neutron source. As many as $10^{9}$ neutrons sr ${ }^{-1}$ were observed in this experiment. Using the deduced proton spectra, neutron generation in lithium, boron, copper, and zinc samples via $(p, n)$ reactions has been simulated and analyzed. It was demonstrated that up to $4 \times 10^{9}$ neutrons sr ${ }^{-1}$ per shot could possibly be generated by ${ }^{7} \mathrm{Li}(p, n)^{7} \mathrm{~B}$ reactions driven by protons accelerated by VULCAN petawatt laser interactions with $\mathrm{Al}$ foil targets. This is greater than an order of magnitude higher than measured by Lancaster et al. ${ }^{25}$ using a similar experimental arrangement on the VULCAN 100-TW laser. It is further encouraging that as the average proton energy increases with increased laser intensity, $(p, n)$ reactions with higher cross sections at higher proton energies (Fig. 5) could be used to further increase the neutron yield from this neutron source. 


\section{ACKNOWLEDGMENTS}

The authors would like to acknowledge the expertise of the VULCAN operations team. One of the authors (J.M.Y.) acknowledges support from China Scholarship Council. (P.McK.) is supported by a Royal Society of Edinburgh/ SEELLD research fellowship. (S.S.) acknowledges the support from the Japan Society for the Promotion of Science. This work is funded by the EPSRC(UK).

${ }^{1} \mathrm{~K}$. Krushelnick (private communication).

${ }^{2}$ J. T. Mendonca, J. R. Davies, and M. Eloy, Meas. Sci. Technol. 12, 1801 (2001).

${ }^{3}$ A. MacKinnon et al., Phys. Rev. Lett. 86, 1769 (2001).

${ }^{4}$ R. A. Snavely et al., Phys. Rev. Lett. 85, 2945 (2000).

${ }^{5}$ I. Spencer et al., Nucl. Instrum. Methods Phys. Res. B 183, 449 (2001).

${ }^{6}$ E. L. Clark et al., Phys. Rev. Lett. 85, 1654 (2000).

${ }^{7}$ P. McKenna et al., Phys. Rev. Lett. 91, 075006 (2003).

${ }^{8}$ P. McKenna et al., Appl. Phys. Lett. 83, 2763 (2003).

${ }^{9}$ P. McKenna et al., Phys. Rev. E 70, 036405 (2004).

${ }^{10}$ K. W. D. Ledingham, P. McKenna, and R. P. Singhal, Science 300, 1107 (2003).
${ }^{11}$ S. Fritzler et al., Appl. Phys. Lett. 83, 3039 (2003).

${ }^{12}$ K. W. D. Ledingham et al., J. Phys. D 37, 2341 (2004).

${ }^{13}$ M. Borghesi et al., Phys. Plasmas 9, 2214 (2002).

${ }^{14}$ K. W. D. Ledingham and P. A. Norreys, Contemp. Phys. 40, 367 (1999).

${ }^{15}$ K. W. D. Ledingham et al., Phys. Rev. Lett. 84, 899 (2000).

${ }^{16}$ I. Spencer et al., Rev. Sci. Instrum. 73, 3801 (2002).

${ }^{17}$ K. W. D. Ledingham et al., J. Phys. D 36, L79 (2003).

${ }^{18}$ J. Magill, H. Schwoerer, F. Ewald, J. Galy, R. Schenkel, and R. Sauerbrey, Appl. Phys. B: Lasers Opt. 77, 387 (2003).

${ }^{19}$ M. I. K. Santala et al., Appl. Phys. Lett. 78, 19 (2001).

${ }^{20}$ J. Yang et al., Appl. Phys. Lett. 84, 675 (2004).

${ }^{21}$ R. F. Barth, A. H. Soloway, J. H. Goodman, R. A. Gahbauer, N. Gupta, T. E. Blue, W. Yang, and W. Tjarks, Neurosurgery 3, 433 (1999).

${ }^{22} \mathrm{http}: / /$ www.act.ac.at/ $\sim$ neutropt/experiments/Radiography

${ }^{23}$ L. J. Perkins et al., Nucl. Fusion 40, 1 (2000).

${ }^{24}$ T. R. Dittrich, B. A. Hammel, C. J. Keane, R. McEachern, R. E. Turner, S. W. Haan, and L. J. Suter, Phys. Rev. Lett. 73, 2324 (1994).

${ }^{25}$ K. L. Lancaster et al., Phys. Plasmas 11, 3404 (2004).

${ }^{26}$ G. Grillon et al., Phys. Rev. Lett. 89, 065005 (2002).

${ }^{27}$ S. Fritzler et al., Phys. Rev. Lett. 89, 165004 (2002).

${ }^{28}$ EXFOR, Cross-section data online at http://www.nea.fr/html/dbdata/x/ welcome.html

${ }^{29}$ Drosg, IAEA-NDS-87 Codes and database for 59 neutron source reactions, 2003. 